\title{
Enabling Calorie-Aware Cooking in a Smart Kitchen
}

\author{
Pei-Yu (Peggy) $\mathrm{Chi}^{1}$, Jen-Hao Chen ${ }^{2}$, Hao-Hua Chu ${ }^{1,2}$, Jin-Ling Lo ${ }^{3,4}$ \\ ${ }^{1}$ Graduate Institute of Networking and Multimedia \\ ${ }^{2}$ Department of Computer Science and Information Engineering \\ National Taiwan University \\ ${ }^{3}$ School of Occupational Therapy, College of Medicine \\ ${ }^{4}$ Department of Physical Medicine and Rehabilitation \\ National Taiwan University Hospital \\ peggychi@csie.org, \{r95922023, haochu, julialo\}@ntu.edu.tw
}

\begin{abstract}
As a daily activity, home cooking is an act of care for family members. Most family cooks are willing to learn healthy cooking. However, learning healthy cooking knowledge and putting the learned knowledge into real cooking practice are often difficult, due to non-trivial nutritional calculation of multiple food ingredients in a cooked meal. This work presents a smart kitchen with UbiComp technology to improve home cooking by providing calorie awareness of food ingredients used in prepared meals during the cooking process. Our kitchen has sensors to track the number of calories in food ingredients, and then provides real-time feedback to users on these values through an awareness display. Our user study suggests that bringing calorie awareness can be an effective means in helping family cooks maintain the healthy level of calories in their prepared meals.
\end{abstract}

Keywords: Ubiquitous Computing / Smart Environments, Home, Healthcare, Context-Aware Computing

\section{Introduction}

After a busy day, many people find nothing better than a delicious home-cooked meal from scratch prepared by a caring family member. This is in accordance with a recent study indicating that most people still favor home-cooked meals or cooking meals from scratch [7]; in Europe, 52\% and the US, 44\% of people prefer scratch cooking. For many family cooks, preparing a tasty meal is as important as a healthy meal with the appropriate amount of calories. However, average family cooks may not know how many calories are in their cooked meals after raw food ingredients are mixed and cooked, or whether these meals are considered healthy and offer a good number of calories for their family members [11]. Reasons include that knowledge on healthy cooking may not be easily learned, and average family cooks cannot easily follow the steps of calculating calories during an intense cooking activity: first they have to estimate accurately the amount (weight) of each food ingredient used (such as oil, meat, vegetables and others), and then they have to look up a food calorie table to calculate and sum up the overall number of calories used in a course or a meal. There- 
fore, they are reluctant to put in much effort on examining and changing their everyday cooking styles. Additionally, a study by Bandura et al. [1] has shown that the expected efforts may cause users to regard their lack of skills and self-efficacy to perform healthy cooking. Based on their theory, increasing accessibility of calorie information to users may raise their confidence and willingness on healthy cooking.

This study presents a Calorie-aware Kitchen that can provide family cooks with awareness on the number of calories in their home cooked meals, thus enhance family cooks' willingness to make healthy meals with the appropriate amount of calories, as recommended by nutritionists. The Calorie-aware Kitchen is augmented with sensors that track the food ingredients used during cooking, and provides just-in-time digital feedback to raise healthy cooking awareness. For instance, when a user prepares a meal, the kitchen presents calorie information whenever the user performs a cooking action that changes the amount of food ingredients on the kitchen counter or the stove, such as by adding meat, pouring in oil, etc. Given the number of calories of each ingredient, an average family cook can perceive calorie information in the amounts of ingredients or the composition of a course. The developed kitchen also suggests the recommended number of calories for a meal, based on the Harris-Benedict equation [10].

\section{Contextual Inquiry}

This work targets experienced family cooks who are willing to cook more healthily but are reluctant to put in much effort on learning and calculating calories. An experienced family cook is defined as someone who can cook without following any recipes or by relying on weight scales to measure food ingredients.

A four-day contextual inquiry was conducted to understand the cooking behaviors of four experienced family cooks (aged 28, 30, 58 and 65) in their home kitchens as they were cooking a regular dinner for their family. During the cooking process, they were observed and videotaped; questions asked about their meal preparation and understanding to nutrition and calorie needs.

Our findings are as follows. (1) They expressed the desire to cook healthily, especially with respect to calorie and nutritional balance. However, given busy schedules, they could not afford too much time or make much effort to learn and follow the complicated steps of weighing food ingredients and calculating nutritional values during actual cooking. They preferred simple-to-understand, practical guidelines for them to refer. (2) Family cooks commonly added ingredients based on experience or preference (oil, butter, meats, for example). Three of them stated that they were unsure about whether their own cooking styles were healthy. (3) Since cooking is an activity that requires ongoing planning and thinking about the next cooking step, family cooks would like to focus solely on cooking. They do not like to be distracted by unrelated activities, such as operating complex electronic interfaces, because distractions are likely to cause cooking errors. They suggested that they want only simple, highly relevant information on cooking itself. (4) They regard a kitchen as part of a home and not a place of work. No standard procedure should tell them how to operate various tools in a kitchen to produce meals. 
The contextual inquiry led to the following design considerations in designing the Calorie-aware Kitchen: (1) the kitchen should offer just-in-time calorie information on food ingredients during their regular cooking process, reduce the effort required to calculate calorie manually, and help family cooks easily perceive calorie information. (2) Calorie recommendations should be provided for ease of comparison and adjustment. (3) Information should help family cooks make their own decisions, without constraining his or her natural cooking habits. When cooks must concentrate, they can choose to ignore the informational display. (4) Information should be presented simply, so that family cooks can easily grasp the calorie information by taking quick glances.

\section{Prototype Design and Implementation}

Based on the above design issues, an initial prototype of the kitchen was proposed, and is presented in Fig. 1(a). The kitchen is comprised of the following two modules; (1) a calorie tracker that tracks the calorie, composition, and position of food ingredients currently on the kitchen counter or stove, and (2) an awareness display that provides calorie information on the ingredients and dishes that mirror actual layout.
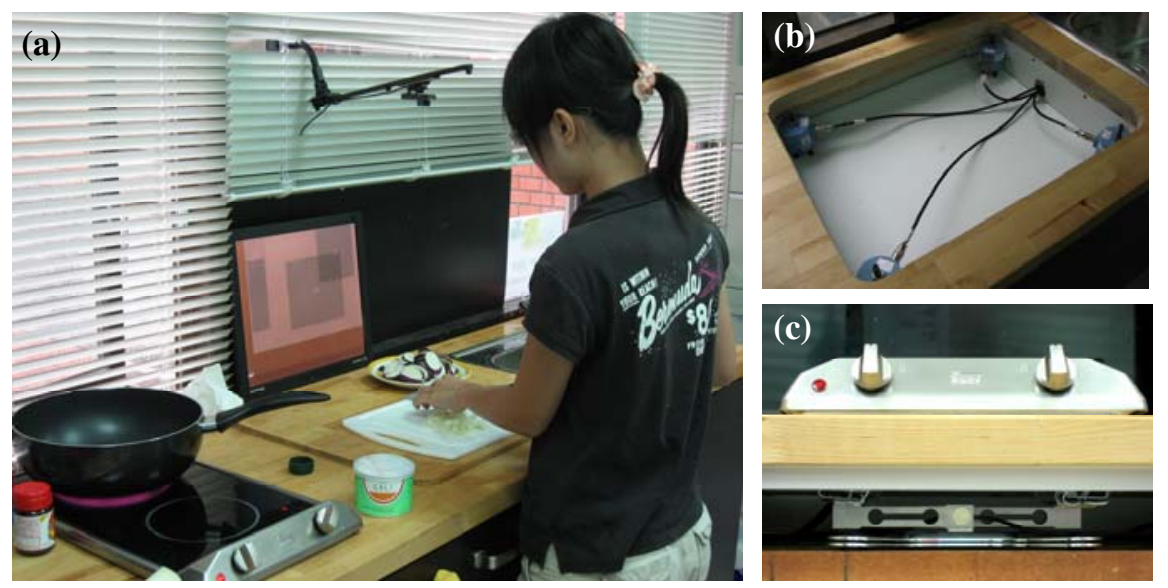

Fig. 1. (a) Calorie-aware Kitchen with digital feedbacks of calorie information during cooking process. An overhead camera is deployed over the counter. Weighing sensors are deployed under counter (b) and stove (c).

\subsection{Calorie Tracker}

Whenever a user performs a cooking action (adding or removing ingredients to or from a container) that may change the number of calories during cooking, the system must detect the cooking action in real-time. An example of such cooking actions is the addition of salad oil (130 kcal) to a pan or the removal of bacon $(250 \mathrm{kcal})$ from a cutting board. Studies have shown that the number of calories can be derived from 
weights of ingredients [17], and calories are additive when composing ingredients. Therefore, to track calories, the weight and the composition of food ingredients in dishes need to be determined.

Our calorie tracker offers a hybrid sensing solution by combining weighing and camera sensing for accurate detection. Fig. 2 depicts the architecture for cooking activity recognition based on hybrid sensing.

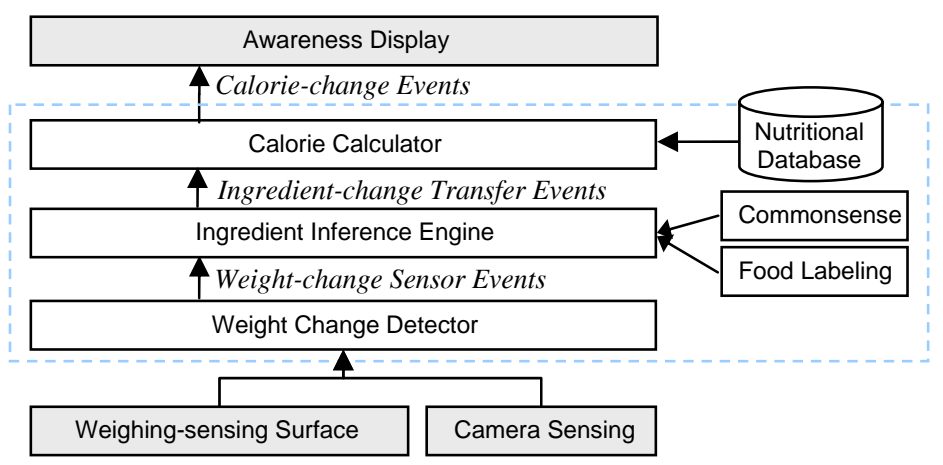

Fig. 2. Calorie tracker architecture.

Hybrid sensing. To calculate calorie in food ingredients, we deployed a weighingsensing surface in the kitchen. Based on our observations of cooking activities, most food preparation activities occur on the kitchen counter. They include putting ingredients on a plate, transferring foods among containers, cutting foods over a cutting board, mixing in a bowl and others. Hence, the system must accurately recognize the amounts (weights) of ingredients that are added to each container to calculate their calories. For the prototype, the design was based on the load sensing table [18] in which four weighing sensors were installed at the four corners underneath the kitchen counter (see Fig. 1(b)). All foods ingredients are assumed to be placed in or on kitchen containers (e.g., plates and bowls, cutting boards are also counted as containers here), rather than being placed directly on the kitchen surface. Hence, the smart counter can track the position of the containers on the countertop with an accuracy of 1 centimeter, and measure the weight of food ingredients in these containers. On the other hand, most cooking activities are performed on the stove, such as frying in a pan, so a weighing sensor must also be present under the stove (Fig. 1(c)). All of the weighing sensors are attached to weight indicators with a resolution of 1 gram, which output readings through a serial port at a frequency of eight samples per second.

Camera sensing using video analysis is employed to improve the accuracy by filtering noise from the weighing-sensing surface. Based on preliminary experiments, detection using only weighing sensors is not sufficiently accurate (recall of 54\%, meaning 46 detections of noise per 100 weight changes), especially when cooking actions, such as cutting or stirring, generate lots of weight noise. Observations indicated that when these actions are performed, the cook performs similar motion of foods using hands and/or utensils. For instance, to cut bacon, the cook uses one hand to hold the bacon and the other hand to take the knife, cutting little by little. Therefore, video analysis using a color histogram comparison $[12,4]$ is performed to filter false 
detections from weighing sensors. We deployed an overhead camera over the counter to capture an overview image of the counter (Fig. 1(a)). By comparing histograms of two camera images captured at different times, it can reduce sensitivity to the motion of objects since unchanging objects differ only slightly in histograms, while a real weight change resulting in large change of color histograms can still be detected.

Cooking activity inference. Our cooking activity inference is based on an eventtriggered system. First, the weight change detector detects Weight-change Sensor Events including weight and position, such as (50 grams, "position:(10, 50)") by processing weight samples from weighing-sensing surface and filtered with camera sensing. Second, an inference rule engine infers ingredient transfer activities by tracking the path of each ingredient from a starting container (as when bacon is put on the cutting board) to an ending container which holds the final cooked meal. A weight matching algorithm similar to that in our earlier work [6] is adopted to track this transference. That is, by matching a weight decrease (such as from a food container on a counter) to a weight increase (such as in a pan on the stove), food ingredient transfer is inferred and an Ingredient-change Transfer Event such as ("container1", "salad oil", 50 grams) is sent to the calorie calculator. Commonsense knowledge on cooking is added to enhance inference engine. For examples, boiling water on the stove produces constant water evaporation resulting in weight decrease, and clams include non-edible shells that has no calorie. Third, because of the difficulties of recognition using computer vision or RFID tags on raw ingredients, a Wizard of $\mathrm{Oz}$ method that involves one human observer's manually inputting the name of an ingredient is currently used to identify new ingredients during cooking process. When the inference engine detects a new ingredient that cannot be inferred by weight matching, the camera captures an image which is then shows to a human observer to ask its name in the other display that the user does not see. A voice-dialog system is also tested to enable family cooks to identify foods using a voice input, for application in the subsequent stage.

Finally, a public nutritional database that provides the nutritional values of each ingredient is used by the calorie calculator to calculate the number of calories, based on the weights and the names of the ingredients [20]. A high-level Calorie-change Event describes ingredients and their calorie amount contained within a container, such as ("container1", "salad oil", $130 \mathrm{kcal}$ ). This value is reported to the awareness display to interact with the user.

Recognition Accuracy and Limitations. From the user study of three participants who cooked a total of 15 meals, our activity recognition accuracy was $92 \%$, meaning that $92 \%$ of the real calorie amounts of ingredients can be determined. For every cooking event (adding/removing ingredients), the average response time is 1 second to show calorie information on the awareness display. Since our tracking method is based on weight matching, the current prototype has a limitation that it cannot recognize concurrent or interleaving events, such as taking two dishes from a counter simultaneously and then immediately putting the ingredients into the pan on the stove. 


\subsection{Awareness Display}

After the calories in ingredients have been determined by the calorie tracker, the system provides real-time feedback, as shown in Fig. 3, to increase the user's awareness of calories via an LCD display on the wall in front of the user.

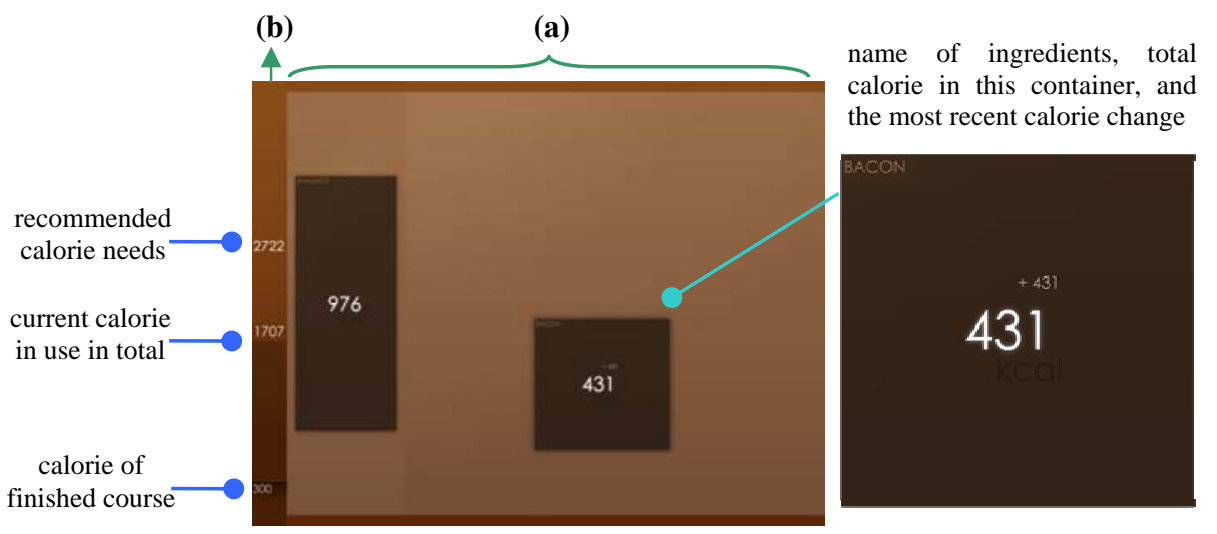

Fig. 3. User interface of Calorie-aware Kitchen, including (a) overview of calorie in the system; (b) recommended calorie needs and current used calories

The main part of this interface gives real-time awareness of calorie in use to users. It presents an overview of the number of calories in current ingredients on the stove and counter (Fig. 3(a)), to enable family cooks to obtain information efficiently. The layout of the information mirrors actual usage and actions, and the information on containers, including the total amount of calories and the names of the ingredients in it, are displayed (Fig. 3 right) based on the real position. All changes on the interface are made with a short and simple sound to notify users.

In the left part of our UI (Fig. 3(b)), a vertical bar is used to show the recommended number of calories for the meal, which is determined using the HarrisBenedict equations (based on weight, height and age) [10]. Therefore, before the system begins, the above details on the user's family members are inputted, and then the system calculates and presents the recommended calorie count for this family. During the cooking process, the current total calories in use are presented, to facilitate comparison for users. Additionally, when a user finishes one course and removes it from the system, the removal is recorded and the number of calories is kept in the bar to reduce users' memory load.

\section{User study}

This section describes the user study. The following two questions guided this study: (1) How effective is the Calorie-aware Kitchen in improving the family cooks' awareness on calories in food ingredients during cooking? (2) What cooking behaviors are affected by the Calorie-aware Kitchen? 
An evaluation was performed to determine how the awareness of calories during cooking affects users. Since the activities in the cooking process are complex, rather than focusing on a specific behavior, a holistic view is taken to gather both quantitative and qualitative observations.

Participants. Three adult participants, P1, P2 and P3 (Table 1), were invited to participate in the user study. They were all experienced cooks of more than five years who regularly cook meals for their family members.

Table 1. Profiles of participants and their family members

\begin{tabular}{cccc}
\hline Participants & P1 & P2 & P3 \\
\hline Age & 24 & 58 & 25 \\
\hline Gender & Female & Female & Male \\
\hline Household size & 4 & 3 & 4 \\
\hline
\end{tabular}

Experimental Design and Procedure. Since our prototype kitchen was constructed in laboratory, it could not be easily moved to each participant's home. Therefore, participants were invited to cook in the laboratory. A video camcorder was used to record the participants' cooking sessions and their interactions with our system; their consent was obtained for subsequent analysis. A concern was raised on whether these participants' cooking behavior would be affected by the presence of the video camcorder (i.e., the "monitor problem" of changing behavior when being watched). Participants expressed that this effect was limited because they already had strong motivation in learning healthy cooking and the presence of video camcorder did not increase/decrease their motivation.

Our user study involved the following three phases: (1) pretest cooking without feedback on calories, (2) test cooking with feedback on calories, and (3) posttest interview. To compare the effectiveness of our smart kitchen between pretest cooking and test cooking phases, each participant was asked to write a fixed dinner menu (Table 2) as if they were to prepare a regular dinner for their family. P1 and P2 wrote a Western dinner menu, whereas P3 wrote a Chinese dinner menu. Based on their dinner menus, they were asked to prepare ingredients using our budget and bring them to our kitchen. Then, the three participants were asked to cook meals in the manner that they did at home, for a total of five cooking sessions per participant in one week. In each cooking session, each participant was asked to cook according to their designated dinner menu in our laboratory kitchen. The participants were given freedom to modify the ingredient composition of the courses (such as by changing the salad dressing, removing mushrooms from spaghetti), but they were not allowed to add a new course or replace an existing course (such as by changing a salad to soup). At the end of the cooking session, participants were free to take their cooked foods home.

In the pretest cooking phase, each participant cooked two meals on two separate days without turning on calorie feedback. Before the start of the first pretest cooking session, the three participants were given time to familiarize with various appliances and the arrangement of cooking tools in the laboratory kitchen. 
Table 2. Menus designed by participants for testing

\begin{tabular}{cl}
\hline Participants & \multicolumn{1}{c}{ Menu } \\
\hline P1 & $\begin{array}{l}\text { Salad (with apple, celery, and thousand-island dressing); Salmon; Fried } \\
\text { aubergine with onion; Spaghetti (with bacon, mushroom, onion, and milk) }\end{array}$ \\
\hline P2 & $\begin{array}{l}\text { New England clam chowder (from Campbell's Condensed Soup [5]); } \\
\text { Bream roll with bacon with special sauce (including UHT whipped cream, } \\
\text { onion, white wine, and lemon), rice and vegetables (cauliflower, carrot, } \\
\text { and sweet corn); Salad (with lettuce and thousand-island dressing) }\end{array}$ \\
\hline P3 & $\begin{array}{l}\text { Shrimp with scrambled egg; Mapo tofu (fried tofu with meat sauce and } \\
\text { green onion); Asparagus with abalone; Chinese Clam Soup; Rice }\end{array}$ \\
\hline
\end{tabular}

In the test cooking phase, participants came to cook for another three meals on three separate days using the calorie feedback on the awareness display. Before the start of the first test cooking session, the calorie feedback interfaces were explained to the participants. The participants were also asked not to perform cooking actions outside the recognition limit of the calorie tracker, i.e., avoid performing concurrent cooking actions. Participants followed this rule with reminders in the first cooking session, and then were able to remember it. Later interviews with participants revealed that although following these rules lengthened the cooking time, it did not affect cooking style.

A posttest interview was performed on the final test cooking day and after the participants finished their last cooking session. They were interviewed about their experience of the kitchen with calorie feedbacks.

Measurement. To determine how effectively participants perceived and utilized calorie awareness information, this study first measured their meal calorie during five cooking sessions. Reduction in meal calories from pretest to test cooking phases suggested that bringing healthy cooking awareness through calorie feedback was effective. The method counted the number of calories in a prepared meal by subtracting the weights of all food ingredients at the end of each cooking session from that at the start of the session. Then, the nutritional database was used to determine the total calories in every meal. Second, the amounts of changes in the ingredients between the pretest and test cooking phases of each participant were analyzed to understand how participants utilized calorie awareness to reduce meal calorie during cooking. Third, the cooking videos were analyzed and coded. The following data were recorded for each cooking session: (1) the frequency with which a participant glanced at the calorie display following a cooking action that resulted in a calorie change, and (2) the average duration of a glance at the awareness display. Finally, the posttest interview involved qualitative measurements of their understanding to ingredients and comments.

\section{Results and Discussion}

Table 3 presents the numbers of meal calories in each cooking session over five days. The two main findings are as follows. All participants reduced the number of meal calories from the pretest cooking phase (without calorie feedback) to the test cooking 
(with calorie feedback) by an average amount of $(195,688,887) \mathrm{kcal}$. All participants cooked meals of calorie count within $\pm 13 \%$ of the recommended amount, and the reduction of calorie used was up to $25.9 \%$. Notably, participant P1 was originally aware of the amounts in use, so the calorie she used in the pretest was already around recommendation (2.8\%). Participants P2 and P3 were lack of nutritional knowledge, and they cooked above the recommended amount during the pretest cooking phase (38.1\% for P2 and $45.6 \%$ for P3). Therefore, the system herein helped them be aware of calories, and further the reduction of meal calories from pretest to test cooking phases was more significant, for P2 (25.9\%) and P3 (22.4\%) than for P1 (6.4\%).

Table 3. Meal calorie (in kcal) during each cooking session

\begin{tabular}{|c|c|c|c|}
\hline Participants & P1 & $\mathbf{P 2}$ & $\mathbf{P 3}$ \\
\hline (1) Recommended calorie & 2,981 & 1,926 & 2,723 \\
\hline Day 1 & 3,070 & 2,677 & 3,951 \\
\hline Day 2 & 3,058 & 2,641 & 3,976 \\
\hline Average & 3,064 & 2,659 & 3,964 \\
\hline Over recommendation & $2.8 \%$ & $38.1 \%$ & $45.6 \%$ \\
\hline Day 3 & 2,937 & 1,916 & 3,308 \\
\hline Day 4 & 2,780 & 2,099 & 3,027 \\
\hline (3) Test & 2,890 & 1,897 & 2,896 \\
\hline Average & 2,869 & 1,971 & 3,077 \\
\hline Over recommendation & $-3.8 \%$ & $2.3 \%$ & $13.0 \%$ \\
\hline (4) Reduction (PretestAVG -TestAVG) & 195 & 688 & 887 \\
\hline Percentage & $6.4 \%$ & $25.9 \%$ & $22.4 \%$ \\
\hline
\end{tabular}

We analyzed how participants changed their cooking behaviors to achieve calorie reduction. Our finding was that our participants were targeting high-calorie ingredients, in which a minor reduction in their amount leads to a significant reduction in the overall meal calories. For instance, in P1's meal, $61.2 \%$ of the total calorie decrease was from the oil. P1 planned to reduce the amount of oil when she found the calorie count was high, and thought it would help keep the number of calories under their required amount, while keeping the meal delicious. In P2's meal, 75.5\% of the total calorie decrease was achieved by reducing the amount of condensed soup. P2 noted that the soup had more calories than she expected, and reducing the amount could greatly lower the calorie count while keeping the meal still tasty. Finally, in P3's meal, $34.8 \%$ of the total calorie decrease was achieved by changing the amounts of meat sauce and tofu. He responded that he found "Mapo Tofu" contained too many calories, so he just used smaller servings to reduce the number of calories.

Table 4 shows the results of video analysis. The first measurement yields the glancing rate, which is defined as the percentage of the times that a participant glanced at the calorie display after a calorie-changing cooking action. A high percentage indicates a strong desire to obtain calorie information. Since the purpose of the kitchen was to promote calorie awareness in users, checking whether users actually checked the calorie display while cooking is important. The glancing rate ranged from 55 to $74 \%$. For instance, P2 was very interested in knowing the number of calories in most ingredients, especially when she put new ingredients on the kitchen surface. The second column in Table 4 lists the average glancing duration, which is defined as the 
average time a participant spends in glancing at the calorie display. A long average duration indicates that users take considerable time to comprehend the calorie information and then make an/no adjustment in the next cooking action. The average duration is about 2 seconds. The analysis indicates that users spent less than 1 second for low-calorie ingredients (such as garlic with $2 \mathrm{kcal}$ ), but more time for high-calorie ingredients (such as spaghetti and oil).

Table 4. Results of video analysis

\begin{tabular}{ccccc}
\hline Participants & P1 & P2 & P3 & Average \\
\hline (1) Glancing rate & $66.7 \%$ & $74.0 \%$ & $55.2 \%$ & $65.3 \%$ \\
\hline (2) Average glancing duration & $2.75 \mathrm{sec}$ & $2.80 \mathrm{sec}$ & $1.48 \mathrm{sec}$ & $2.34 \mathrm{sec}$ \\
\hline
\end{tabular}

The findings of the posttest interviews are described below. P1 said, "After perceiving this information, I would also consider the amounts of ingredients in my shopping. For example, now I have ideas about buying the appropriate size of salmon (given calorie consideration), and I will be careful not to buy (food ingredients) beyond my calorie target." P2 stated that "This kind of instant feedback is effective to remind me of what I already know about using the condensed soup and some highcalorie ingredients such as UHT cream." P3 said, "I'm glad to get this kind of calorie information without additional effort, because I should really be aware of using less of an (high-calorie) ingredient and not all in the whole package."

Participants had the following expectations of the future direction of this kitchen: (1) they were interested in preparing a nutritional balanced meal, including appropriate servings covering all five major groups of foods (grains, vegetables, fruits, milk, and meat and beans). However, nutritional balance is difficult to measure, record, and understand. (2) They wanted expert cooking tips, during their cooking sessions, about healthy alternatives or substitutes for certain less-healthy food ingredients (e.g., olive oil as a substitute of butter) or cooking method (e.g., frying).

\section{Related Work}

Work and commercial products have exploited mobile devices or ubiquitous computing to record personal food intake and calories, and further persuade people into healthy behaviors. MyFoodPhone [15] is a nutrition tracking service running on mobile phones, allowing users to send pictures of consumed foods, get feedbacks from dietitian, and share their record with the community. This provides a new method of engagement. The Diet-aware Dining Table [6] can track what and how much users eat on the dining table and then provide nutritional awareness to diners. Work from Mankoff et al. [14] tracks nutrition of foods users have taken and provides suggestions about healthier foods based on analysis of shopping receipt data. However, these projects focus on tracking and recording food intake itself; therefore differ from our work, which focuses on raising calorie awareness on preparing and cooking foods in home kitchens.

Much research effort has focused on augmenting kitchens with various digital media to create rich, interactive experiences for users cooking in a kitchen. Some work 
has focused on increasing awareness to support multi-tasking activities in the kitchen. For instance, the Counter Intelligence project from MIT [3] augmented a kitchen with ambient interfaces to improve the usability of the physical environment. It assists users to determine temperatures, find things, follow recipes and time steps during meal preparation. Other work has focused on digital interactive recipes that guide users through a step-by-step cooking process. For instance, Hamada et al. [9] developed a recipe navigation system that provides just-in-time instruction with multimedia information including text, video, and audio. Such digital recipes offer a more interactive experience than a paper-based recipe book. Terrenghi et al. [19] presented the Living Cookbook, which enabled people to share cooking experience with others, to teach cooking lessons to others, and thus to foster of social relations. Rather than augmenting kitchens with a range of digital media to create interactive cooking experiences, our smart kitchen focuses on promoting healthy cooking by raising nutritional awareness during the cooking process, while leaving the decision about how to cook to the users.

Some related projects targeted cooking activity or food ingredient recognition. The Intelligent Kitchen project [16] presented an activity recognition system that adopted data mining techniques to infer what would be the next human cooking action and offer suggestion on the next cooking step through an LCD display or a robot. Kranz et al. [13] developed an augmented cutting board and knife to infer the type of food being handled. Bolle et al. [2] developed a vision-based system that recognized different types of fruits and vegetables. Commercial calorie scales [8] allow users to weight foods, identify names manually, and check their calories. Our work differs from them in tracking ingredients by an augmented kitchen during the cooking process, and show real-time calorie awareness for users to perceive.

\section{Conclusion and Future Work}

The Calorie-aware Kitchen employs UbiComp technology to improve traditional meal preparation and cooking by raising awareness of calorie information in ingredients that go into a meal. The kitchen is augmented with sensors to track ingredients and calorie changes during the cooking process, and then provides digital feedback on calories. The user study result suggested that providing just-in-time calorie awareness to users during their cooking process can be an effective mean in helping these family cooks maintain the healthy level of calories in their prepared meals.

For our future work, our smart kitchen will consider a broader context in its social and culture impacts of using UbiComp technology to promote healthy cooking. Cooking should be considered as a social behavior involving both meal preparation and consumption. Therefore, our design and user study should cover not only family cooks but also feedbacks on taste from family members as meal consumers. Finally, in addition to conducting a longer and larger-scale user study, a comparison between our design and traditional education should be made. This will enable us to observe different ways people could use this calorie information while preparing different dinner menus and help us to clarify the impacts of our system. 


\section{References}

1. Bandura, A.: Social Foundations for Thought and Action: A Social Cognitive Theory. NJ: Prentice Hall, Englewood Cliffs (1986)

2. Bolle, R. M., Connell, J., Haas, N., Mohan, R., Taubin G.: VeggieVision: A Produce Recognition System. In: Proc. WAIAT-97: 1997 IEEE Workshop on Automatic Identification Advanced Technologies, pp. 35--38 (1997)

3. Bonanni, L., Lee, C.-H., Selker, T.: Attention-based Design of Augmented Reality Interfaces. In: Ext. Abstracts CHI 2005, pp. 1228--1231. ACM Press (2005)

4. Boreczky, J.S., Rowe, L.A.: Comparison of Video Shot Boundary Detection Techniques. In: Proc. SPIE: Storage and Retrieval for Still Image and Video Databases IV, vol. 2670 (1996)

5. Campbell Soup Company. Campbell's Condensed Soup, http://www.campbellsoup.com/condensed_soups.asp (2007)

6. Chang, K.-H., Liu, S.-Y., Chu, H.-H., Hsu, J., Chen, C., Lin, T.-Y., Huang, P. Diet-aware Dining Table: Observing Dietary Behaviors over Tabletop Surface. In: Proc. PERVASIVE 2006: the 4th international conference on Pervasive Computing, pp. 366--382 (2006)

7. Datamonitor Co. Changing Cooking Behaviors \& Attitudes: Beyond Convenience, Commercial Report (2006)

8. Digiweigh Scale, http://www.digiweighusa.com/ (2007)

9. Hamada, R., Okabe, J., Ide, I., Satoh, S., Sakai, S., Tanaka, H.: Cooking Navi: Assistant for daily cooking in kitchen. In: Proc. MM2005: the 13th ACM International Multimedia Conference, pp. 371--374. ACM Press (2005)

10. Harris, J., Benedict, F.: A Biometric Study of Basal Metabolism in Man. Washington D.C. Carnegie Institute of Washington (1919).

11. INSIGHT 20. Consumption of Food Group Servings: People's Perception vs. Reality. In: USDA Center for Nutrition Policy and Promotion (2000).

12. Koprinska, I., Carrato, S.: Temporal Video Segmentation: a Survey. In: Signal Processing: Image Communication, vol. 16, pp. 477--500 (2001)

13. Kranz, M., Schmidt, A., Maldonado, A., Rusu, R. B., Beetz, M., Hörnler, B., Rigoll, G.: Context-Aware Kitchen Utilities. In: adjunct Proc. TEI 2007: 1st Intl. Conf. on Tangible and embedded Interaction, pp. 213--214 (2007),

14. Mankoff, J., Hsieh, G., Hung, H. C., Lee, S., Nitao, E.: Using Low-Cost Sensing to Support Nutritional Awareness. In: Proc. Ubicomp 2002: the 4th international conference on Ubiquitous Computing (2002)

15. MyFoodPhone Nutrition Inc. MyFoodPhone, http://www.myfoodphone.com/ (2007).

16. Nakauchi, Y., Fukuda, T., Noguchi, K., Matsubara, T.: Intelligent Kitchen: Cooking Support by LCD and Mobile Robot with IC-Labeled Objects. In: Proc. IROS 2005: International Conference on Intelligent Robots and Systems, pp. 1911--1916 (2005)

17. National Public Health Institute (KTL), Finland. Collected Information on National Trends and Databases of Composite Foods and Industrial Ingredients. In: EuroFIR Partners, European Food Information Resource Network (2005)

18. Schmidt, M., Strohbach, K., Van Laerhoven, A. Friday, H.-W. Gellersen.: Context Acquisition based on Load Sensing. In: Proc. Ubicomp 2002: the 4th international conference on Ubiquitous Computing (2002)

19. Terrenghi, L., Hilliges, O., Butz, A.: Kitchen Stories: Sharing Recipes with the Living Cookbook. In: Personal and Ubiquitous Computing, vol. 11, pp 409--414 (2006)

20. United States Department of Agriculture. Nutrient Data Products and Services, http://www.ars.usda.gov/Services/docs.htm?docid=13725 